\title{
riccafd
}

Revista Iberoamericana de Ciencias de la Actividad Física y el Deporte

\section{EFECTOS DE LA TAREA DUAL SOBRE EL TIRO LIBRE EN BALONCESTO}

\section{EFFECTS OF THE DUAL TASK ON THE FREE THROW IN BASKETBALL}

Collado-Mateo, D. ${ }^{1,2}$; Villafaina, S.1; Fernández-González, E. ; Gozalo, M.'; Fuentes, J.P. ${ }^{1}$ y Gusi, N. ${ }^{1}$

${ }^{1}$ Facultad de Ciencias del Deporte, Cáceres (España). dcolladom@unex.es; svillafaina@unex.es; efernanddo@alumnos.unex.es; mgozalo@unex.es;.jpfuent@unex.es; ngusi@unex.es

${ }^{2}$ Facultad de Educación, Universidad Autónoma de Chile, Talca, Chile.

Correspondencia: Collado-Mateo, D._dcolladom@unex.es

Código UNESCO / UNESCO code: 5899 Educación Física y Deportiva / Physical Education and Sports.

Classificación Consejo de Europa / Council of Europe classification: 12. Aprendizaje motor / Motor learning

Recibido el 6 de febrero de 2019

Aceptado el 26 de abril de 2019

DOI: http://dx.doi.org/10.24310/riccafd.2019.v8i2.6447

Agradecimientos: El autor DCM es beneficiario de una beca del Ministerio Español de Educación, Cultura y Deporte (FPU14/01283). En este sentido, el autor SV es beneficiario de un contrato predoctoral cofinanciando por el Fondo Social Europeo y la Consejería de Economía e Infraestructura de la Junta de Extremadura (PD16008).

Los financiadores no han participado en la recogida de datos, análisis, decisión de publicar o preparación del manuscrito.

\section{RESUMEN}

El objetivo de este estudio fue estudiar los efectos de una tarea dual en el tiro libre en baloncesto, comparando esa influencia en función de la categoría y el sexo de los jugadores. Los resultados muestran que existe un descenso del rendimiento provocado por la tarea dual de los baloncestistas de categoría infantil y junior $(p<0,05)$, no observándose lo mismo en la categoría senior. Tampoco se detectaron diferencias significativas entre el rendimiento de hombres y mujeres. Puede afirmarse, por tanto, que las tareas duales provocan un descenso en el rendimiento, sobre todo en etapas de formación, posiblemente por la automatización del patrón motor debido a un mayor tiempo de práctica en categorías senior. Por ello, las tareas duales pueden ser incluidas en los entrenamientos 
para crear situaciones estresantes y bajo presión similares a las que se pueden encontrar los deportistas en situaciones competitivas reales.

Palabras clave: tiro libre, baloncesto, tarea dual, aprendizaje motor.

\section{ABSTRACT}

The objective was to study the effects of a dual task in the free throw in basketball, comparing that influence according to the category and the gender of the players. Results show that there is a decrease in performance caused by the dual-task of the junior and junior players $(p<0.05)$, not observing the same in the senior category. No significant differences were detected between the performance of men and women. For these reasons, dual-tasks may cause a decrease in performance, especially in training stages, possibly due to the automation of the motor pattern caused by a longer practice time in senior categories. Therefore, dual tasks can be included by coaches in training to create stressful situations under pressure similar to those that athletes can find in real competitive situations.

Key words: free throw, basketball, dual-task, motor learning.

\section{INTRODUCCIÓN}

El tiro libre es un aspecto técnico de dominio indispensable para todos los jugadores de baloncesto, independientemente de la categoría de juego y del puesto específico que desempeñen (1). Entre un $20-25 \%$ de los puntos totales de un equipo en un partido son anotados por medio del tiro libre (1). Como ejemplo de este hecho, durante la temporada 2015/2016 en la NBA, Minnesota Timberwolves anotó más de 21 tiros libres por partido, siendo el equipo que más anotaba de esta manera, al contrario que Orlando Magic, que anotó apenas 15 puntos por partido desde la línea de tiro libre. Además, en cuanto a porcentajes de acierto, estos oscilaron entre el $66,8 \%$ y el $80,5 \%$ que anotaron Detroit Pistons y New York Knicks, respectivamente. En general, cada equipo dejó de meter aproximadamente entre 5 y 9 puntos por partido por errar tiros libres. A pesar de ello, existen algunos estudios que aseguran que el tiro libre no es un factor significativo para ganar partidos y que apenas existe una relación significativa si el partido tiene un marcador ajustado. En definitiva, según Fierro Hernández (2) la relevancia del tiro libre a lo largo de un partido es mayor que si se realiza a lo largo de una temporada.

Diferentes factores condicionan el rendimiento en el tiro libre. Lidor \& Arnon (3) ya mencionaban diferentes factores, distinguiendo entre psicológicos (capacidad de superar barreras mentales como la ansiedad, la motivación o el miedo), sociológicos (cohesión del equipo, liderazgo...) o factores fisiológicos (fuerza, fatiga...). El momento de efectuar el tiro libre es otro factor relevante, siendo diferente si este se produce al principio o al final del partido, así como el marcador en ese preciso momento ya que el porcentaje de acierto en momentos críticos 
(finales "apretados") oscilan con respecto al resto del partido (4). La posición del jugador suele ser importante en este contexto. En la temporada 2015/2016, los pívots (centers) fueron los que peor porcentaje de acierto obtuvieron en la NBA, con un $70,1 \%$ de acierto medio, mientras que los bases-escoltas (guards) fueron los más efectivos con un $81,27 \%$ de acierto. Además, la edad del jugador, su experiencia deportiva y si va ganando o va perdiendo el partido en ese instante también serán aspectos importantes a tener en cuenta (5).

Se puede afirmar que el porcentaje de puntos anotados de tiros libres y el porcentaje de efectividad de tiro libre aumenta según aumenta la categoría y por tanto la edad de los sujetos (6). Con la edad, los jugadores adquieren y automatizan patrones técnicos y se produce un cambio y un desarrollo físico, haciendo que la efectividad en la acción del tiro libre sea mayor. Según algunos estudios, en el caso de los hombres existe una diferencia significativa entre 13-14 años y los 15-16 en adelante. Por otro lado, en el caso de las mujeres parece que donde existe un cambio significativo a nivel de efectividad es desde los 11-12 años y los 13-14 (7).

La ejecución del tiro libre está, por tanto, condicionada por numerosos factores. El lanzador debe intentar disminuir todos los efectos negativos que pudieran causar los factores contextuales y psicológicos, así como focalizar su atención en la propia ejecución de su tiro libre. Un modo de evaluación y entrenamiento de la focalización de la atención es la conocida como "tarea dual", en la que el sujeto debe realizar una acción cognitiva o motora mientras realiza simultáneamente otra acción secundaria cognitiva o motora. El objetivo de este tipo de tareas es el de comprobar cómo se divide la atención en el conocido como "paradigma compartido"(8). Existe algún estudio en el cual se trabajaba aplicando al tiro libre diversas tareas simultáneamente y evaluando una serie de factores (9), señalando la relevancia de la rutina previa al lanzamiento. En otros deportes, como el béisbol, también se ha señalado que la cantidad de atención dedicada a la tarea secundaria estará inversamente relacionada con el nivel de rendimiento en la tarea primaria (10).

Hasta la fecha, no tenemos constancia de la existencia de estudios en las principales bases de datos que comparen los resultados obtenidos en una prueba con tarea dual entre distintas categorías ni sexos, por lo que sería importante esta evaluación para saber posibles diferencias entre sujetos de distintas edades.

El objetivo de este estudio es comprobar los efectos de una tarea dual en el tiro libre en baloncesto, comparando esa influencia en función de la categoría y el sexo de los jugadores.

\section{MÉTODO}

\section{Diseño}

Se trató de un estudio observacional, descriptivo y de corte transversal, según la clasificación de Manterola \& Otzen (11). El estudio fue aprobado por el comité de bioética de la Universidad de Extremadura ( $n^{\circ}$ registro: 04/2018). Todos los 
participantes firmaron el consentimiento informado para participar en el estudio. En el caso de los sujetos menores de edad, se informó a los padres o tutores y ellos fueron quiénes firmaron el mencionado consentimiento.

\section{Participantes}

Los participantes del estudio fueron un total de 47 sujetos, cada uno de los cuales lanzó un total de 40 tiros libres, siendo el total de registros 1880 . Del total de la muestra, 24 fueron hombres y 23 mujeres, divididos en 6 equipos de diferente categoría, siendo 3 de ellos femeninos y 3 masculinos. En total hay 8 jugadores masculinos en categoría infantil, 8 en categoría junior y 8 en categoría senior. En la categoría femenina fueron analizadas 8 jugadoras infantiles, 8 en categoría junior y 7 en categoría senior. A excepción del equipo senior masculino, el total del resto de equipos son del equipo Miralvalle (categoría femenina), y Fundación Cintra Plasencia (categoría masculina), ambos conjuntos de Plasencia. Los jugadores senior masculinos son componentes del Adepla Gastrobar Cáparra, equipo militante en la Primera División Extremeña.

\section{Procedimiento}

En primer lugar, cada participante realizó un calentamiento de 10 minutos, siendo el mismo de las mismas características al de cada sesión de entrenamiento. Las pruebas se realizaron en el pabellón habitual de entrenamiento en medio de una sesión de entrenamiento táctica. A continuación respondieron a un cuestionario sociodemográfico en el que se realizaron una serie de preguntas respecto a su edad, rendimiento (máximo nivel competitivo, lesiones recientes y años de experiencia). Tras lo anterior, se llevó a cabo la prueba.

Se diferenciaron dos tareas: 1) lanzamiento de 20 tiros libres simples y 2) lanzamiento de 20 tiros libres mientras, simultáneamente, se realizaba una cuenta hacia atrás desde 100 hasta 0 , sucesivamente de 3 en 3 . En un estudio revisado se establecía una cuenta atrás de 2 en 2 y de 7 en 7 (12). En el presente estudio se optó por una opción intermedia y que la cuenta atrás fuese de 3 en 3 (13).

Los jugadores fueron agrupados por parejas, de tal manera que mientras uno realizaba la tarea, el otro se encargaba de recoger el balón y devolvérselo al lanzador. Para evitar el posible efecto de empezar con los tiros libres con o sin tarea secundaria, la mitad de los jugadores efectuaron primero 20 tiros libres simples y luego 20 tiros libres con la tarea dual, mientras que la otra mitad lo haría al revés. En cuanto al gesto técnico de lanzar a canasta, se pidió al jugador que si tenía algún tipo de rutina previa al lanzamiento la llevase a cabo, pues diversos estudios han comprobado que el acierto es mayor cuando se realizan las rutinas preliminares, mejorando, entre otros, la concentración previa al lanzamiento (14-16).

Para la realización de la cuenta atrás por parte de los lanzadores, se reprodujo un sonido que definía intervalo temporal de 2 segundos que los jugadores debían tomar como ritmo para ir haciendo la cuenta atrás. Tanto en la tarea simple como en la tarea dual el método de puntuación se estableció siguiendo el ejemplo del 
estudio de Price et al. (9) y dependió de si el balón no tocaba aro (0 puntos), si el balón golpeaba en el borde (1 punto), o el balón era encestado (2 puntos). Por último, finalizada la prueba se les pidió una valoración sobre la dificultad de las tareas.

\section{Análisis estadístico}

En cuanto al análisis estadístico, en primer lugar se realizaron las pruebas de Kolmogorov-Smirnov y de Shapiro-Wilk, para comprobar si las variables analizadas seguían una distribución normal. Con el fin de comparar el rendimiento con tarea dual y sin tarea dual, se realizó una prueba $T$ para muestras relacionadas, dividiendo la muestra por categorías. A continuación se realizó un ANOVA de un factor para comparar entre las tres categorías estudiadas (infantiles, juniors y seniors). Finalmente, se realizó una prueba T para muestras independientes con el objetivo de realizar una comparación entre sexos.

\section{RESULTADOS}

De acuerdo con los resultados obtenidos en las pruebas de KolmogorovSmirnov y Shapiro-Wilk, se optó por utilizar una estadística paramétrica, ya que las variables principales cumplían con las hipótesis de normalidad.

Como puede observarse en la tabla 1, tanto en la categoría infantil como en la categoría junior se aprecian diferencias significativas $(p<0,05)$ a la hora de comparar los aciertos en la prueba sin y con la aplicación de la tarea dual. Si se compara la media sin tarea dual con la media con tarea dual vemos de manera clara que hay una diferencia mayor entre las categorías infantil y junior que en la categoría senior. Así pues, observando las diferencias en los resultados obtenidos por infantiles y juniors en la prueba sin tarea dual $(26,50$ y 30,94) y, comparándola, con los resultados obtenidos en la prueba con tarea dual en estas mismas categorías $(28,69$ y 33,00$)$, puede afirmarse hay un descenso del rendimiento en estas dos categorías. Sin embargo, en los resultados obtenidos en la categoría senior $(p=0,114)$ no se aprecian diferencias significativas.

Tabla 1: Influencia de la tarea dual en las diferentes categorías.

\begin{tabular}{llllllll}
\hline \multirow{2}{*}{\begin{tabular}{l} 
Variable \\
\cline { 3 - 7 }
\end{tabular}} & Grupo & $\mathrm{N}$ & \multicolumn{2}{l}{ Lanzamiento sin tarea dual } & $\begin{array}{l}\text { Lanzamiento } \\
\text { dual }\end{array}$ & con tarea \\
\cline { 3 - 7 } & & & Media & DT & Media & DT & P-valor \\
\hline \multirow{4}{*}{ Aciertos } & Infantil & 16 & 28,69 & 3,911 & 26,50 & 2,828 & 0,028 \\
& Junior & 16 & 33,00 & 3,386 & 30,94 & 4,986 & 0,005 \\
& Senior & 15 & 34,67 & 3,559 & 33,47 & 2,800 & 0,114 \\
\hline
\end{tabular}

DT: Desviación típica

En la tabla 2 pueden observarse los resultados obtenidos en cada categoría en cada una de las variables analizadas. En cambio, en la tabla 1 lo que se 
aprecia es si había diferencias entre la prueba con y sin tarea dual sin comparar resultados entre ninguna de las tres categoría. En la tabla 2 se observa como al hacer esa comparación entre las tres categorías se encuentran diferencias significativas $(p<0,05)$ en varias de las variables analizadas. En primer lugar y por orden de importancia se aprecian diferencias significativas si se atiende a los resultados obtenidos tanto en la prueba con tarea dual como en la prueba sencilla. Se constata como los jugadores de la categoría senior obtuvieron mejores resultados en ambas pruebas $(33,47$ y 34,67$)$ respectivamente que los jugadores de la categoría junior $(30,94$ y 33,00$)$ e infantil $(26,50$ y 28,69$)$. Además se encuentran también diferencias significativas atendiendo a los años de experiencia de los sujetos. Sin embargo, atendiendo a cómo perciben los sujetos de las distintas categorías la dificultad de la tarea secundaria (tarea cognitiva) y la dificultad de ambas tareas no se encuentran diferencias significativas.

Tabla 2: ANOVA de las variables analizadas en función de la categoría

\begin{tabular}{|c|c|c|c|c|}
\hline Variable & Grupo & $\mathrm{N}$ & Media \pm DT & P-valor \\
\hline & Infantil & 16 & $12,88 \pm 0,34$ & \\
\hline \multirow[t]{3}{*}{ Edad } & Junior & 16 & $17,06 \pm 0,93$ & $<0,001$ \\
\hline & Senior & 15 & $24,67 \pm 4,50$ & \\
\hline & Infantil & 16 & $5,44 \pm 1,21$ & \\
\hline \multirow[t]{3}{*}{ Años Experiencia } & Junior & 16 & $8,13 \pm 2,06$ & $<0,001$ \\
\hline & Senior & 15 & $14,07 \pm 4,91$ & \\
\hline & Infantil & 16 & $26,5 \pm 2,83$ & $<0.001$ \\
\hline \multirow[t]{3}{*}{ Acierto con tarea dual $(0 ; 1 ; 2)$} & Junior & 16 & $30,94 \pm 4,99$ & \\
\hline & Senior & 15 & $33,47 \pm 2,80$ & \\
\hline & Infantil & 16 & $28,69 \pm 3,91$ & \\
\hline \multirow[t]{3}{*}{ Acierto sin tarea dual $(0 ; 1 ; 2)$} & Junior & 16 & $33 \pm 3,39$ & $<0,001$ \\
\hline & Senior & 15 & $34,67 \pm 3,56$ & \\
\hline & Infantil & 16 & $3,5 \pm 2,07$ & \\
\hline \multirow[t]{3}{*}{ Dificultad tarea secundaria $(1-10)$} & Junior & 16 & $3,69 \pm 1,99$ & $<0,001$ \\
\hline & Senior & 15 & $3,67 \pm 1,59$ & \\
\hline & Infantil & 16 & $7,56 \pm 1,41$ & \\
\hline \multirow[t]{2}{*}{ Dificultad ambas (1-10) } & Junior & 16 & $6,69 \pm 1,45$ & $<0,001$ \\
\hline & Senior & 15 & $6,53 \pm 1,30$ & \\
\hline
\end{tabular}

DT: Desviación típica.

Si se atiende a la tabla 3 se aprecian los datos y resultados obtenidos en la comparación por sexos en cada una de las variables analizadas. Se aprecia como existen diferencias significativas $(p<0,05)$ en cuanto a los aciertos con la aplicación de la tarea dual, observandose un mayor acierto en hombres $(31,74)$ que en mujeres $(28,79)$. Además también se aprecian diferencias significativas en como los sujetos perciben la dificultad de ambas tareas, es decir, las mujeres perciben la tarea con más dificultad $(7,46)$ que los hombres $(6,39)$. Por el con- 
trario, no se observan diferencias significativas en el resto de variables, ni en el acierto sin la tarea dual, en la dificultad de la tarea secundaria ni, sobre todo, en la pérdida de rendimiento tal y como hemos comentado anteriormente.

En cuanto a la comparación por sexos, no se aprecian diferencias significativas a la hora de comparar la pérdida de rendimiento entre sexos $(p=0,094)$. Entendemos como pérdida de rendimiento la comparación de las diferencias existentes entre los resultados obtenidos en la prueba sin tarea dual y la prueba con tarea dual en ambos sexos, es decir, como podemos ver en la tabla 4, los hombres tuvieron un resultado de 32,83 y las mujeres 31,33 en la prueba sin tarea dual. En la prueba con tarea dual los resultados fueron de 31,74 y 28,79 respectivamente. La diferencia o pérdida de rendimiento en hombres fue de 1,09 y en mujeres fue de 2,54. Comparando estos datos no puede afirmarse que haya una mayor pérdida de rendimiento en mujeres con respecto a hombres.

Tabla 3: Datos estadísticos en la comparación por sexos

\begin{tabular}{lllll}
\hline Variables & Sexo & $\mathrm{N}$ & Media DT & P-valor \\
\hline Acierto con tarea dual $(0 ; 1 ; 2)$ & Hombre & 23 & $31,74 \pm 5,09$ & \multirow{2}{*}{0,280} \\
& Mujer & 24 & $28,79 \pm 3,72$ & \\
\hline Acierto sin tarea dual $(0 ; 1 ; 2)$ & Hombre & 23 & $32,83 \pm 5,01$ & 0,253 \\
& Mujer & 24 & $31,33 \pm 3,00$ & \\
\hline Dificultad tarea secundaria & Hombre & 23 & $3,26 \pm 1,86$ & \multirow{2}{*}{0,202} \\
& Mujer & 24 & $3,96 \pm 1,83$ & \\
\hline Dificultad ambas & Hombre & 23 & $6,39 \pm 1,23$ & \multirow{2}{*}{0,009} \\
& Mujer & 24 & $7,46 \pm 1,44$ & \\
\hline Perdida de rendimiento & Hombre & 23 & $1,09 \pm 2,78$ & \multirow{2}{*}{0,094} \\
& Mujer & 24 & $2,54 \pm 3,04$ & \\
\hline
\end{tabular}

DT: Desviación típica

\section{DISCUSIÓN}

El presente estudio investiga los efectos de la tarea dual en el tiro libre en baloncesto, comparando esa influencia en función de la categoría y el sexo de los jugadores. Analizando los resultados observamos que, en la muestra estudiada, existe un descenso del rendimiento de los jugadores de categoría infantil y junior $(p<0,05)$, no observándose lo mismo en la categoría senior.

En este sentido, los jugadores de categoría senior tienen mejores resultados que los jugadores de categoría junior y éstos a su vez que los jugadores infantiles. Esto está relacionado de manera significativa con, obviamente, la edad de los sujetos y además de manera directamente proporcional con los años de 
experiencia de dicho sujeto (17). Esto lo podemos comprobar en los datos de la tabla 2 y comprobar cómo, a pesar de que en todas las categorías perciben de manera similar la dificultad de la tarea secundaria, obtienen resultados diferentes. Al hilo de lo anteriormente expuesto también confirmamos la hipótesis que enuncia que, si bien a medida que se sube de categoría, la efectividad en el tiro libre será mayor, vemos un salto importante entra la categoría infantil y junior, siendo esta diferencia mayor que en comparación con el salto entre juniors y seniors, como podemos observar en la tabla 1. Estos resultados apoyarían el modelo de los tres estadios propuestos por Fitts \& Posner (18) para el aprendizaje motor. En este sentido, nuestros resultados muestran como los jugadores senior, con un mayor acierto a canasta, tienen más automatizado el patrón motor, derivado de un mayor tiempo de práctica (19).

En cuanto a la comparación por sexos, tomando como referencia a Zuzik (7) preveíamos que no habría diferencias entre hombres y mujeres, y analizando los resultados obtenidos se confirma que no hay diferencias significativas y por tanto las mujeres no tienen una mayor pérdida de rendimiento que los hombres, como se puede apreciar en la tabla 3 . Atendiendo a los resultados de la tabla 4 hacemos referencia a diferencias significativas $(p<0,05)$ en el acierto con la tarea dual en hombres, cuyos resultados son mayores que en las mujeres, pero al no encontrar diferencias en la pérdida de rendimiento no hace a este hecho algo muy relevante. Por último, a pesar de no encontrar bibliografía existente que comparase la pérdida de rendimiento en una tarea dual entre hombres y mujeres vemos en los resultados de la tabla 4 que obtenemos un p-valor de 0,09, por tanto determinamos que no existen diferencias.

En definitiva los atletas que tengan un mejor desempeño en presencia de la tarea secundaria, es posible que ante situaciones estresantes o de mucha presión, tengan mejores respuestas. En esta línea, los atletas con peor desempeño en presencia de la tarea secundaria demuestran que, en su caso particular, la interferencia entre la tarea primaria y secundaria es mayor, por lo que puede que requieran más práctica en las situaciones sencillas de una sola tarea en su deporte en concreto o un entorno de tarea dual menos complicada. En relación con lo anterior, Chen et al. (20), en la modalidad de taekwondo, demostraron como los deportistas de nivel élite tenían mejores condiciones en cuanto a tiempo de reacción y más posibilidades de anticipar el movimiento del rival.

En este sentido, investigaciones centradas en estudiar la resiliencia en el deporte, muestran como la concentración es uno de los aspectos más importantes que diferencian a los atletas de élite (21-23). Por ello, entrenamientos que planteen tareas duales, podrían ser muy interesantes para el entrenamiento de la concentración, simulando una situación estresante y con presión similar a la que se encontrarían en una situación de competición real, provocando fatiga mental. Estudios previos como el de Alarcón López et al. (24) muestran que los jugadores fatigados mentalmente destacan por obtener unos peores resultados en los tiros libres. En esta línea, Cárdenas Vélez et al. (25) sostiene que esta fatiga mental es bastante usual en los jugadores de baloncesto, ya que se desenvuelven en un entorno con un nivel muy elevado de entropía que les obliga a realizar constantes operaciones mentales para dar respuesta a las demandas de 
la tarea, lo que supone un enorme esfuerzo mental. Por lo tanto, como comentamos anteriormente, este tipo de tareas, donde a una tarea motora se le incluye de manera simultánea una cognitiva, podría ser de enorme utilidad en el campo del entrenamiento.

\section{Limitaciones}

En primer lugar destacar que la muestra no ha sido excesivamente grande $(\mathrm{N}=47)$, lo cual podría limitar la obtención de resultados significativos. En este sentido, el estudio de Gabbett \& Abernethy (26) encontró que jugadores profesionales de rugby seniors reducían su rendimiento al incluir una tarea dual, lo que refuerza la suposición de que de haber tenido una muestra mayor habríamos encontrado diferencias en todas las categorías. En segundo lugar, comentar que entre nuestros participantes, nos encontramos con jugadores con niveles de juego diferentes. A la hora de agruparlos en subgrupos nos encontramos con 7 jugadores nivel élite (han jugado en primeras ligas o selecciones internacionales), con unos 15 jugadores de nivel nacional (han competido en ligas a nivel nacional o en campeonatos con selecciones autonómicas) y con unos 25 jugadores de nivel regional (han participado en ligas a nivel autonómico). Lo ideal sería haber tenido una muestra con jugadores que estén en un nivel competitivo similar y haber podido comparar los resultados de una manera más objetiva.

\section{CONCLUSIONES}

En este estudio y con la muestra analizada, se encontró una pérdida de rendimiento significativa cuando se añadió una tarea cognitiva a una motora en jugadores de baloncesto de categoría infantil y junior. Sin embargo, en jugadores senior no se observa esta pérdida de rendimiento, lo que puede estar relacionado con una automatización del patrón motor debido a un mayor tiempo de práctica y, por tanto, mayor nivel de especialización.

Por lo anterior, las tareas duales podrían ser incluidas en los entrenamientos como un factor positivo tendente a crear situaciones estresantes y bajo presión similares a las que pueden encontrarse los deportistas en situaciones competitivas reales.

\section{REFERENCIAS}

1. Vélez DC, Rojas J. Determinación de la incidencia del tiro libre en el resultado final a través del análisis estadístico. European Journal of Human Movement. 1997(3):17786.

2. Fierro Hernández $C$. Variables relacionadas con el éxito deportivo en las ligas NBA y ACB de baloncesto. Revista de Psicología del Deporte. 2002;11(2):0247-255.

3. Lidor R, Arnon M. Correlational relationships between technical variables and final placing of basketball teams. Coaching and Sport Science Journal. 1997;2(2):39-47. 
4. Navarro RM, Calvo AL, Ruano MAG, Sampaio J. Analysis of critical moments in the league ACB 2007-08. Revista de Psicología del Deporte. 2009;18(3):391-5.

5. Oñoro Asenjo MÁ, Gómez Ruano MÁ, Jiménez Saíz S, Calvo AL. Análisis del drop en baloncesto a través del estudio de los tiros libres. Kronos. 2015;14(2).

6. Sampaio J, Fraga F, Silva T, editors. La evolución de las estadísticas de los tiros libres en partidos de baloncesto de formación. III Congreso de la Asociación Española de Ciencias del Deporte; 2004.

7. Zuzik P. Free throw shooting effectiveness in basketball matches of men and women. Sport Science Review. 2011;20(3-4):149-60.

8. Huang H-J, Mercer VS. Dual-Task Methodology: Applications in Studies of Cognitive and Motor Performance in Adults and Children. Pediatric Physical Therapy. 2001;13(3):133-40.

9. Price J, Gill DL, Etnier J, Kornatz K. Free-throw shooting during dual-task performance: Implications for attentional demand and performance. Research Quarterly for Exercise and Sport. 2009;80(4):718-26.

10. Prezuhy AM, Etnier JL. Attentional patterns of horseshoe pitchers at two levels of task difficulty. Research Quarterly for Exercise and sport. 2001;72(3):293-8.

11. Manterola C, Otzen T. Estudios Observacionales: Los Diseños Utilizados con Mayor Frecuencia en Investigación Clínica. International Journal of Morphology. 2014;32:634-45.

12. Laing JM, Tokuno CD. The effects of dual-tasking on arm muscle responses in young and older adults. Human movement science. 2016;46:159-66.

13. Brustio PR, Magistro D, Rabaglietti E, Liubicich ME. Age $\square$ related differences in dual task performance: A cross $\square$ sectional study on women. Geriatrics \& Gerontology International. 2016.

14. Ajamil DL, Pascual JG, Idiakez JA, Echevarría BG. Análisis de patrones temporales en las rutinas gestuales previas al tiro libre de baloncesto, en la categoría alevín. Revista de Psicología del Deporte. 2011;20(2):383-400.

15. Larumbe Zabala E. Entrenamiento de variables psicológicas para los tiros libres. Revista de Psicología del Deporte. 2001;10(1):0089-97.

16. Le Lobmeyer D, Wasserman E. Preliminaries to free throw shooting: Superstitious behavior. Journal of Sport Behavior. 1986;9(2):70.

17. Gallahue DL, Ozmun J, Goodway J. Understanding motor development: infants, children, adolescents, adults (ed.): New York, McGraw-Hill; 2002.

18. Fitts PM, Posner MI. Human performance. 1967.

19. Magill RA, Anderson DI. Motor learning and control: Concepts and applications: McGraw-Hill New York; 2007.

20. Chen C-Y, Jing Dai I, Chen F, Chou K-M, Chang C-K. Reliability and validity of a dualtask test for skill proficiency in roundhouse kicks in elite taekwondo athletes. Journal of Sports medicine. 2015;6:181.

21. Sarkar M, Fletcher D. Psychological resilience in sport performers: a review of stressors and protective factors. Journal of Sports Sciences. 2014;32(15):1419-34.

22. Moran AP. The psychology of concentration in sport performers: A cognitive analysis: Psychology Press; 2016. 
23. Fletcher D, Sarkar M. A grounded theory of psychological resilience in Olympic champions. Psychology of Sport and Exercise. 2012;13(5):669-78.

24. Alarcón López F, Ureña Ortín N, Cárdenas Vélez D. La fatiga mental deteriora el rendimiento en el tiro libre en baloncesto. Revista de Psicología del Deporte. 2017;26(3):0033-36.

25. Cárdenas Vélez D, Conde-González J, Perales JC. El papel de la carga mental en la planificación del entrenamiento deportivo. Revista de Psicología del Deporte. 2015;24(1):0091-100.

26. Gabbett TJ, Abernethy B. Dual-task assessment of a sporting skill: influence of task complexity and relationship with competitive performances. Journal of Sports Sciences. 2012;30(16):1735-45. 